\title{
Contribution of CYP3A Isoforms to Dealkylation of PDE5 Inhibitors: A Comparison between Sildenafil $N$-Demethylation and Tadalafil Demethylenation
}

\author{
Rikako Takahiro, ${ }^{a}$ Saki Nakamura, ${ }^{a}$ Hiroyuki Kohno, ${ }^{a}$ Naoki Yoshimura, ${ }^{b}$ Tsuneyuki Nakamura, \\ Sayaka Ozawa, ${ }^{d}$ Keiichi Hirono, ${ }^{d}$ Fukiko Ichida, ${ }^{d}$ and Masato Taguchi*, ${ }^{a}$ \\ ${ }^{a}$ Graduate School of Medicine and Pharmaceutical Sciences, University of Toyama; 2630 Sugitani, Toyama 930- \\ 0194, Japan: ${ }^{b}$ First Department of Surgery, Faculty of Medicine, University of Toyama; 2630 Sugitani, Toyama \\ 930-0194, Japan: ${ }^{c}$ Department of Pediatrics, Kanazawa Medical University; 1-1 Daigaku Uchinada-cho, Kahoku- \\ gun, Ishikawa 920-0293, Japan: and ${ }^{d}$ Department of Pediatrics, Faculty of Medicine, University of Toyama; 2630 \\ Sugitani, Toyama 930-0194, Japan.
}

Received August 5, 2014; accepted October 7, 2014

\begin{abstract}
The aim of this study was to characterize the kinetics of metabolite formation of the phosphodiesterase type-5 (PDE5) inhibitors sildenafil and tadalafil by CYP3A4, CYP3A5, and CYP3A7 isoforms. The formations of $\mathrm{N}$-desmethyl sildenafil and desmethylene tadalafil were examined using CYP3A supersomes co-expressing human P450 oxidoreductase and cytochrome $b_{5}$. Both sildenafil $N$-demethylation and tadalafil demethylenation were catalyzed by CYP3A4, CYP3A5, and to a lesser extent by CYP3A7. The kinetics of desalkyl metabolite formation of the two drugs were well fitted to the Hill equation; however, the Hill coefficients (n) suggested CYP3A-mediated negative cooperativity. Next, we analyzed the kinetics with a two binding sites model assuming two reaction steps: reaction 1 with high-affinity and low-capacity metabolism and reaction 2 with low-affinity and high-capacity metabolism. The kinetics of desalkyl metabolite formation were also fitted to the two binding sites model. The intrinsic clearance $\left(C L_{\text {int }}\right)$ values of reactions 1 and 2 for sildenafil $N$-demethylation were 0.733 and $0.033 \mu \mathrm{L} / \mathrm{min} / \mathrm{pmol} P 450$ for CYP3A4, 0.788 and $0.019 \mu \mathrm{L} / \mathrm{min} / \mathrm{pmol}$ P450 for CYP3A5, and 0.079 and $0.004 \mu \mathrm{L} / \mathrm{min} / \mathrm{pmol}$ P450 for CYP3A7, respectively. The $C L_{\text {int }}$ values of reactions 1 and 2 for tadalafil demethylenation were 0.187 and $0.014 \mu \mathrm{L} / \mathrm{min} / \mathrm{pmol}$ P450 for CYP3A4, 0.050 and $<0.001 \mu \mathrm{L} / \mathrm{min} / \mathrm{pmol} \mathrm{P} 450$ for CYP3A5, and 0.004 and $<0.001 \mu \mathrm{L} / \mathrm{min} / \mathrm{pmol} \mathrm{P} 450$ for CYP3A7, respectively. These results may provide the basis not only for understanding the metabolic properties of the two PDE5 inhibitors, but also for one possible explanation of the mechanisms of CYP3A-mediated negative cooperativity.
\end{abstract}

Key words CYP3A; sildenafil; tadalafil; negative cooperativity; two binding sites model

Phosphodiesterase type-5 (PDE5) is one of the key enzymes hydrolyzing cyclic guanosine monophosphate (cGMP) in vascular smooth muscle. ${ }^{1)}$ PDE5 inhibitors have been shown to be an effective vasodilator, and have been used widely as a treatment not only for erectile dysfunction, but also for pulmonary arterial hypertension (PAH) ${ }^{2)}$ Sildenafil and tadalafil are orally active inhibitors of PDE5 that are approved for the treatment of PAH in Japan, but their half-life period in the body differs substantially.,4) That is, sildenafil has a fairly short half-life of about $4-5 \mathrm{~h}$, whereas tadalafil has a long half-life of about $17.5 \mathrm{~h}$ in healthy subjects. ${ }^{5,6)}$ The short half-life of sildenafil makes it the drug of choice in patients with more severe cardiovascular disease, allowing early use of supportive treatment if an adverse clinical event occurs. ${ }^{7)}$ In contrast, tadalafil has the advantage of once a day dosing compared to sildenafil's three times a day dosing, with implications for patient convenience and compliance. ${ }^{6,8}$ Therefore, a transition from sildenafil to tadalafil has been frequently tried in stable patients with $\mathrm{PAH} .^{3,8)}$

In humans, sildenafil is eliminated predominantly by hepatic metabolism and is converted to an active metabolite, $N$-desmethyl sildenafil, with properties similar to the parent drug. ${ }^{9)}$ The $N$-demethylation by CYP3A is the major route of metabolism of sildenafil in humans ${ }^{10)}$ (Fig. 1). Recently, $\mathrm{Ku}$ et al. ${ }^{11)}$ showed that both CYP3A4 and CYP3A5 played a significant role in the metabolism of sildenafil using CYP3A supersomes. That is, the intrinsic clearance for $N$-dealkylation of sildenafil by CYP3A5 and CYP3A4 were 0.09 and $0.07 \mu \mathrm{L} /$ $\mathrm{min} / \mathrm{pmol} \mathrm{P} 450$, respectively. ${ }^{11)}$ They also demonstrated that the mean rate for $N$-desalkyl metabolite formation from sildenafil was high in human liver microsome preparations with CYP3A5 activity (heterozygous for $C Y P 3 A 5^{*} 1 / * 3$ alleles) compared to those with null CYP3A5 activity (homozygous for $C Y P 3 A 5^{*} 3$ allele). ${ }^{11)}$ These results suggested that genetic polymorphism of CYP3A5 at least partly contributes to interindividual variability in the disposition of sildenafil. On the other hand, tadalafil is eliminated predominantly by oxidative metabolism to a catechol via demethylenation (Fig. 1), which then undergoes methylation and glucuronidation to form the major inactive metabolite, a methylcatechol glucuronide. ${ }^{12)}$ The oxidative metabolism of tadalafil is mainly mediated by cytochrome P450 (CYP) 3A in the human liver. ${ }^{13,14)}$ However, it is unclear whether CYP3A4 and CYP3A5 share the metabolic pathway of tadalafil because no systematic study on the CYP3A isoforms involved in the demethylenation of the drug has been reported in the literature to date.

In addition to CYP3A4/5, CYP3A7 is known to be a major $\mathrm{P} 450$ isoform highly expressed in the fetal liver, ${ }^{15,16)}$ and a shift in hepatic CYP3A expression from CYP3A7 to CYP3A4 occurs after birth. ${ }^{15)}$ The expression level of CYP3A7 is significant through 6 months postnatal age, ${ }^{16)}$ and it is also detectable in low amounts in some adult livers. ${ }^{17)}$ Given that both 
<smiles>CCCC1NN(C)c2c1nc(-c1cc(S(=O)(=O)N3CCN(C)CC3)ccc1OCC)[nH]c2=O</smiles>

Sildenafil

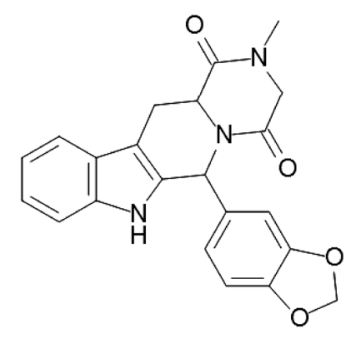

Tadalafil<smiles>CCCC1NN(C)c2c1nc(-c1cc(S(=O)(=O)N3CCNCC3)ccc1OCC)[nH]c2=O</smiles>

$N$-Desmethyl sildenafil

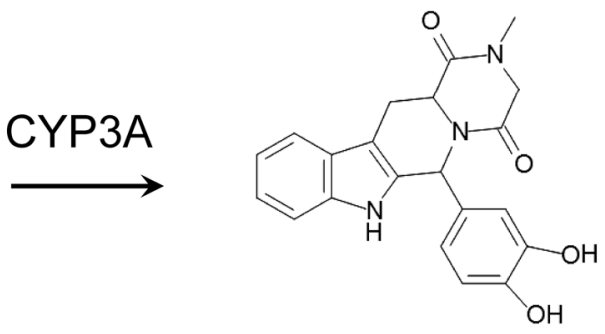

Desmethylene tadalafil

Fig. 1. The Metabolic Pathways of Sildenafil and Tadalafil in Human Liver Microsomes

sildenafil and tadalafil are frequently administered to neonates and/or infant patients with PAH associated with congenital heart disease, basic information on the comparison of the kinetics of CYP3A7-catalyzed metabolism may be of interest to physicians treating children with these drugs.

The primary objective of this study was to characterize the metabolic properties of CYP3A4, CYP3A5, and CYP3A7 in the formation of metabolites from sildenafil and tadalafil by using CYP3A supersomes co-expressing human P450 oxidoreductase (POR) and cytochrome $b_{5}$. The Hill equation was used for kinetic analysis, and the results indicated the negative cooperative properties of $\mathrm{P} 450$ enzymes in the dealkylation of the two PDE5 inhibitors. Thus, we evaluated the fittings for metabolite formation rate versus substrate concentration curves with a two binding sites model.

\section{MATERIALS AND METHODS}

Materials Sildenafil, $N$-desmethyl sildenafil, tadalafil, desmethylene tadalafil, and vardenafil were purchased from Toronto Research Chemicals (Toronto, Canada). tert-Butyl methyl ether was purchased from Nacalai Tesque (Kyoto, Japan). Cyclopentyl methyl ether was purchased from Wako Pure Chemical Industries, Ltd. (Osaka, Japan). Microsomes from baculovirus-infected insect cells expressing human CYP3A, POR and cytochrome $b_{5}$ (Supersomes ${ }^{\circledR}$, Lot number 2276593 for CYP3A4; Lot number 2130704 for CYP3A5; Lot number 77694 for CYP3A7) were purchased from BD Gentest (Woburn, MA, U.S.A.). All other chemicals and solvents were of the highest purity available.

Metabolism of Sildenafil and Tadalafil by Expressed CYP Enzymes Metabolism of sildenafil and tadalafil was tested with CYP3A supersomes, and the formation rates of $\mathrm{N}$-desmethyl sildenafil and desmethylene tadalafil were determined. The reaction mixture containing CYP3A enzyme and nicotinamide adenine dinucleotide phosphate (NADPH) in $50 \mathrm{~mm}$ potassium phosphate buffer $(\mathrm{pH}$ 7.4) was preincubated for $5 \mathrm{~min}$ at $37^{\circ} \mathrm{C}$. The reaction was started by adding $100 \mu \mathrm{L}$ of pre-warmed sildenafil or tadalafil solution containing $0.4 \%$ bovine serum albumin to $100 \mu \mathrm{L}$ of reaction mixture. The reaction mixture (total volume, $200 \mu \mathrm{L}$ ) was incubated for $10 \mathrm{~min}$ at $37^{\circ} \mathrm{C}$ in a shaking water bath. The final concentrations of CYP3A and NADPH in the incubation medium were $20 \mathrm{pmol} / \mathrm{mL}$ and $2 \mathrm{~mm}$, respectively. The reaction was stopped by adding $100 \mu \mathrm{L}$ of ice-cold acetonitrile and immediately placing the mixture on ice. For all the experiments, sildenafil and tadalafil were dissolved in methanol and acetonitrile to the required concentrations, respectively. The concentration of organic solvent did not exceed $2 \%$ in the final reaction mixture. Pilot experiments were performed with each biotransformation and CYP3A form to ensure that comparisons of the metabolite formation by CYP3A4, CYP3A5, and CYP3A7 were determined under linear rate conditions.

$\mathrm{N}$-Desmethyl Sildenafil Assay Concentrations of $\mathrm{N}$-desmethyl sildenafil were determined by using a Thermo Fisher Accela LC system (Thermo Fisher Scientific, Yokohama, Japan) coupled to an LTQ-Orbitrap XL ETD system (Thermo Fisher Scientific). Sample preparation was performed using of liquid-liquid extraction as described previously ${ }^{18,19)}$ with a minor modification. That is, a $200 \mu \mathrm{L}$ aliquot of reaction sample was mixed with $2 \mathrm{~mL}$ of $0.1 \mathrm{M}$ glycine buffer (saturated with $\mathrm{NaCl}, \mathrm{pH} 10.6$ ), and extracted with $5 \mathrm{~mL}$ of tert-butyl methyl ether. Four $\mathrm{mL}$ of the organic phase was then evaporated to dryness with a SpeedVac ${ }^{\circledR}$ system (Savant, Famingdale, NY, U.S.A.). The residue was reconstituted in $200 \mu \mathrm{L}$ of the mobile phase containing vardenafil as an internal standard $^{20)}$ and $10 \mu \mathrm{L}$ was injected into the system.

Chromatographic separation of $\mathrm{N}$-desmethyl sildenafil was done using an InertSustain ${ }^{\circledR} \mathrm{C} 18$ column $(15 \mathrm{~cm} \times 2.1 \mathrm{~mm}$ i.d.; $3 \mu \mathrm{m}$ particle size; GL Sciences, Tokyo, Japan). The mobile 
phase was $10 \mathrm{~mm}$ ammonium formate and acetonitrile $(40: 60$, $\mathrm{v} / \mathrm{v})$ that contained $0.1 \%$ formic acid. The flow rate of the mobile phase was $300 \mu \mathrm{L} / \mathrm{min}$, and the column temperature was $40^{\circ} \mathrm{C}$. To identify the metabolites, mass spectra were recorded by electrospray ionization in the positive mode. The detector was operated in selected reaction monitoring (SRM) mode using the transitions of $N$-desmethyl sildenafil at $\mathrm{m} / \mathrm{z}$ $461.0 \rightarrow 283.0$ and vardenafil at $489.1 \rightarrow 151.0$, respectively. ${ }^{20)}$ The peak areas were calculated using Qualbrowser software (Thermo Fisher Scientific). Since the assay was linear between 0.02 and $10 \mu \mathrm{M}$, samples were diluted appropriately to keep the drug concentration within the range. The coefficients of variation (CV) for $N$-desmethyl sildenafil was $6.52 \%$ and $5.32 \%$ for concentrations of $2 \mu \mathrm{M}$ and $10 \mu \mathrm{M}$, respectively.

Desmethylene Tadalafil Assay Concentrations of desmethylene tadalafil in reaction mixtures were determined by a reversed-phase HPLC method, as described by Farthing et $a .^{21)}$ with a minor modification. That is, the total reaction mixture was mixed with $2 \mathrm{~mL}$ of $1 \mathrm{M}$ citrate buffer (saturated with $\mathrm{NaCl}, \mathrm{pH} 4.5$ ), and extracted with $5 \mathrm{~mL}$ of cyclopentyl methyl ether. The organic phase was then evaporated to dryness with a SpeedVac ${ }^{\circledR}$ system (Savant, Famingdale, NY, U.S.A.). The residue was reconstituted in $200 \mu \mathrm{L}$ of the mobile phase and $50 \mu \mathrm{L}$ was injected into the HPLC system.

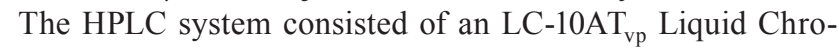
matograph Series (Shimadzu, Kyoto, Japan) with a model RF-20 A fluorescence detector (Shimadzu) and an Inertsil ${ }^{\mathbb{R}}$ ODS-3 column $(15 \mathrm{~cm} \times 4.6 \mathrm{~mm}$ i.d.; $3 \mu \mathrm{m}$ particle size; GL Sciences). The mobile phase was $10 \mathrm{~mm}$ ammonium formate and acetonitrile $(50: 50, \mathrm{v} / \mathrm{v})$ that contained $0.1 \%$ formic acid. The flow rate of the mobile phase was $0.65 \mathrm{~mL} / \mathrm{min}$, and the column temperature was $40^{\circ} \mathrm{C}$. Peaks were monitored at an excitation wavelength of $275 \mathrm{~nm}$ and an emission wavelength of $335 \mathrm{~nm},{ }^{21)}$ and the retention time was $3.7 \mathrm{~min}$ for desmethylene tadalafil. The quantitation limit for desmethylene tadalafil was $1 \mathrm{nM}$ and the $\mathrm{CV}$ for desmethylene tadalafil was $5.13 \%$ and $0.85 \%$ for concentrations of $5 \mathrm{~nm}$ and $50 \mathrm{~nm}$, respectively.

Kinetic Analysis The kinetic parameters for sildenafil $N$-demethylation and tadalafil demethylenation by each CYP enzyme were estimated using a Hill equation (Eq. 1) for simplicity:

$$
v=\frac{V_{\max } \cdot S^{n}}{K_{\mathrm{m}}+S^{n}}
$$

where $v$ is the velocity of the reaction, $S$ is the substrate concentration, $K_{\mathrm{m}}$ is the substrate concentration at which $v$ is $50 \%$ of the maximum $V_{\max }$, and $n$ is the Hill coefficient as a qualitative measure of the degree of cooperativity. ${ }^{11,22)}$ Intrinsic clearance $\left(C L_{\text {int }}\right)$ for sildenafil $N$-demethylation and tadalafil demethylenation by CYP3A4, CYP3A5, and CYP3A7 was calculated using Eq. 2.

$$
C L_{\mathrm{int}}=\frac{V_{\max }}{K_{\mathrm{m}}}
$$

The kinetic parameters for sildenafil $N$-demethylation and tadalafil demethylenation by CYP3A4, CYP3A5, and CYP3A7 were also estimated using the two binding sites $\operatorname{model}^{23)}$ as follows:

$$
v=\left(\frac{V_{\max 1} \cdot S}{K_{\mathrm{m} 1}}+\frac{V_{\max 2} \cdot S^{2}}{K_{\mathrm{m} 1} \cdot K_{\mathrm{m} 2}}\right) /\left(1+\frac{S}{K_{\mathrm{m} 1}}+\frac{S^{2}}{K_{\mathrm{m} 1} \cdot K_{\mathrm{m} 2}}\right)
$$

The hypothesis of this model is that two substrates can bind simultaneously within the active site of a P450 enzyme. This model involves two sets of parameters, $K_{\mathrm{m} 1}$ and $V_{\max 1}$, for the initial binary enzyme-substrate complex and $K_{\mathrm{m} 2}$ and $V_{\max 2}$ for the ternary enzyme-substrate complex at high substrate concentrations. ${ }^{22,23)}$ When $S \ll K_{\mathrm{m} 2}$ and $K_{\mathrm{m} 1} \ll K_{\mathrm{m} 2}$, the equation for a two binding sites model is given as in Eq. $4{ }^{23)}$

$$
v=\left(\frac{V_{\max 1} \cdot S+\left(V_{\max 2} / K_{\mathrm{m} 2}\right) \cdot S^{2}}{K_{\mathrm{m} 1}+S}\right)
$$

These kinetic models were fitted with nonlinear leastsquare regression using NONMEM software (double precision NONMEM Version V Level 1.1, PREDPP Version IV Level 1.1, and NM-TRAN Version III Level 1.1 running on a personal computer using Windows 7).

\section{RESULTS}

Sildenafil $\mathrm{N}$-demethylation was catalyzed by CYP3A4 and CYP3A5 (Figs. 2A, B), similar to the previous report by $\mathrm{Ku}$ et $a{ }^{11)}$ It was also indicated that CYP3A7 could catalyze sildenafil $\mathrm{N}$-demethylation, although the metabolite formation was slow (Fig. 2C). The kinetics of $N$-desmethyl sildenafil formation by CYP3A isoforms were well fitted to the Hill equation (Fig. 2). The estimated $K_{\mathrm{m}}$ values for CYP3A4, CYP3A5, and CYP3A7 isoforms were 27.7 $\pm 7.5,17.4 \pm 3.2$, and $107 \pm 21 \mu \mathrm{M}$, respectively, and the $V_{\max }$ values for CYP3A4, CYP3A5, and CYP3A7 isoforms were $30.7 \pm 11.3,18.9 \pm 6.1$, and $9.92 \pm 0.01 \mathrm{pmol} / \mathrm{min} / \mathrm{pmol} \mathrm{P} 450$, respectively (Table 1). The Hill coefficients $(n)$ for CYP3A4, CYP3A5 and CYP3A7 were $0.64 \pm 0.11,0.71 \pm 0.19$, and $0.52 \pm 0.05$, respectively, suggesting negative cooperativity (Table 1 ).

Next, we also evaluated the kinetics of desmethylene metabolite formation from tadalafil by CYP3A4, CYP3A5, and CYP3A7 (Fig. 3). The tadalafil demethylenation was catalyzed by CYP3A4 and to a lesser extent by CYP3A5. CYP3A7 could also catalyze tadalafil demethylenation (Fig. 3). These metabolite formation rates were considerably lower compared with sildenafil (Fig. 2). The kinetics of desmethylene tadalafil formation were well fitted to the Hill equation (Fig. 3). The $K_{\mathrm{m}}$ values for CYP3A4, CYP3A5, and CYP3A7 isoforms were $5.29 \pm 0.47,7.76 \pm 1.05$, and $171 \pm 68 \mu \mathrm{M}$, respectively, and the $V_{\max }$ values were $0.658 \pm 0.048,0.467 \pm 0.069$, and $0.872 \pm 0.221 \mathrm{pmol} / \mathrm{min} / \mathrm{pmol} \mathrm{P} 450$, respectively (Table 1). The Hill coefficients $(n)$ for CYP3A4, CYP3A5, and CYP3A7 were $0.60 \pm 0.05,0.72 \pm 0.13$, and $0.66 \pm 0.06$, respectively, suggesting negative cooperativity (Table 1 ).

To highlight the observed negative cooperativity of the CYP3A-mediated dealkylation process (Table 1), a visual inspection of the Eadie-Hofstee plot was used for data presentation (Figs. 2, 3). A biphasic plot was clearly observed in sildenafil $N$-demethylation by CYP3A7 (Fig. 2C) and tadalafil demethylenation by CYP3A4 (Fig. 3A). These results suggested that the dealkylation metabolism consisted of two components, one with low-affinity and one with high-affinity. Since each dealkyl reaction was catalyzed by the single expressed $\mathrm{P} 450$ protein, it was considered reasonable to assume 
(A)

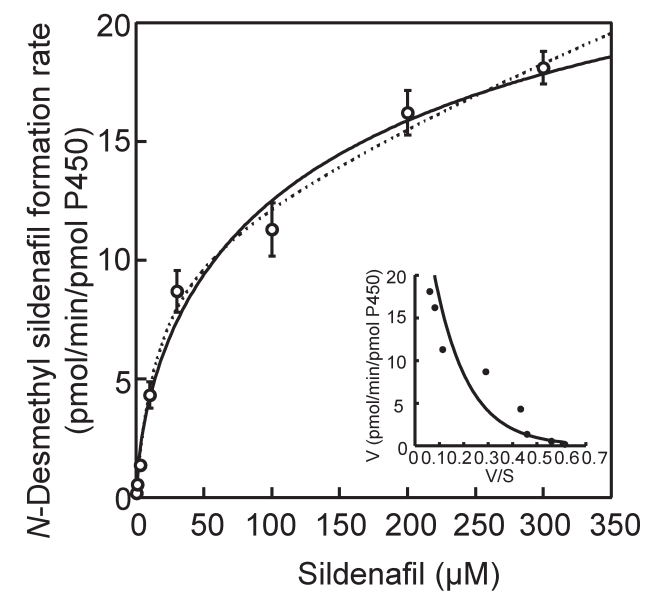

(B)

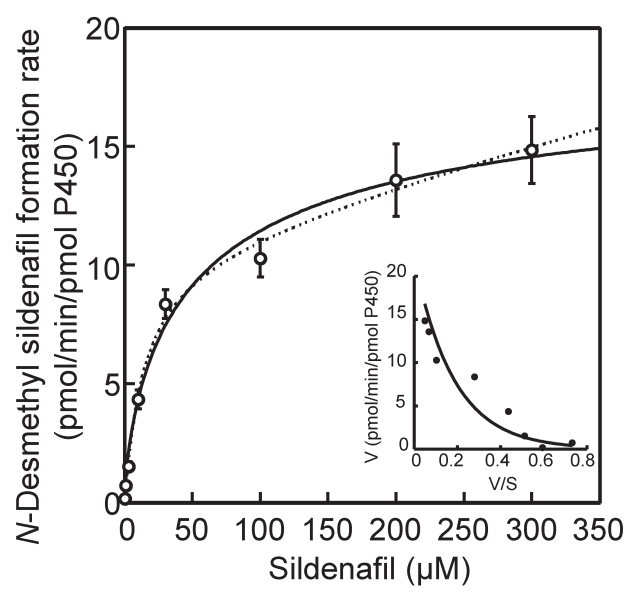

(C)

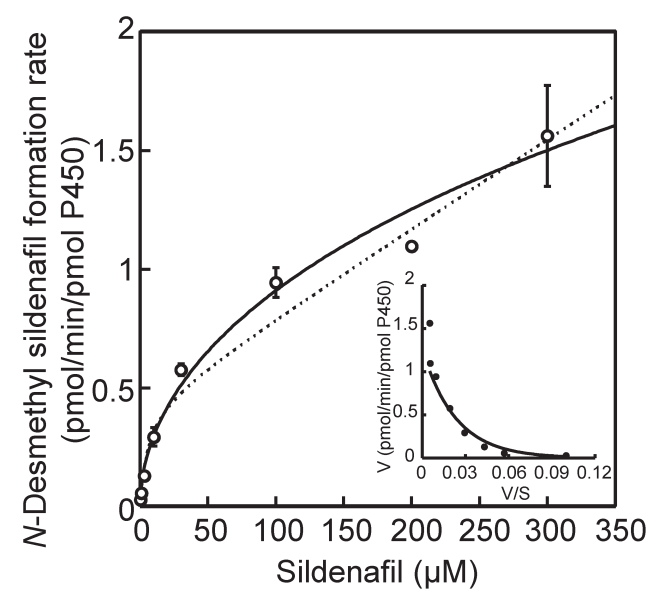

Fig. 2. The Kinetics of $N$-Desmethyl Sildenafil Formation from Sildenafil by CYP3A4 (A), CYP3A5 (B), and CYP3A7 Isoforms (C)

Various concentrations of substrate $(0-300 \mu \mathrm{m})$ were incubated with recombinan human CYP3A and NADPH at $37^{\circ} \mathrm{C}$ for $10 \mathrm{~min}$. The velocity $(\mathrm{pmol} / \mathrm{min} / \mathrm{pmol}$ $\mathrm{P} 450)$ versus sildenafil concentration was fitted to sigmoidal model (solid line) and two binding sites model (dotted line). The corresponding Eadie-Hofstee plot (velocity versus velocity/substrate concentration) is shown in the inset. Each point represents average \pm S.E. $(n=4)$.

that there were at least two binding sites in the P450 enzyme. In addition, it means little to compare the metabolite kinetics of sildenafil and tadalafil based on the parameters listed in Table 1 because the exponent for the Hill equation $(n \neq 1)$ has no direct relationship to $K_{\mathrm{m}}$ and $V_{\max }$ values. ${ }^{22)}$ Therefore, for further consideration regarding negative cooperativity in the CYP3A-mediated dealkylation process, we performed data
(A)

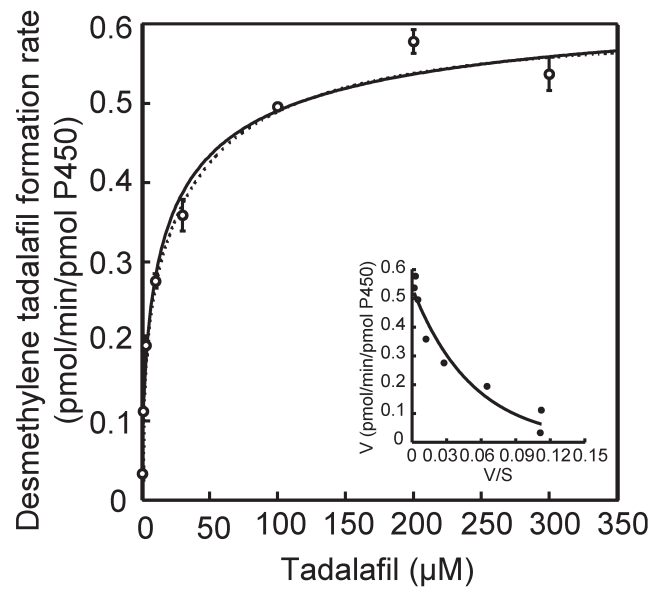

(B)

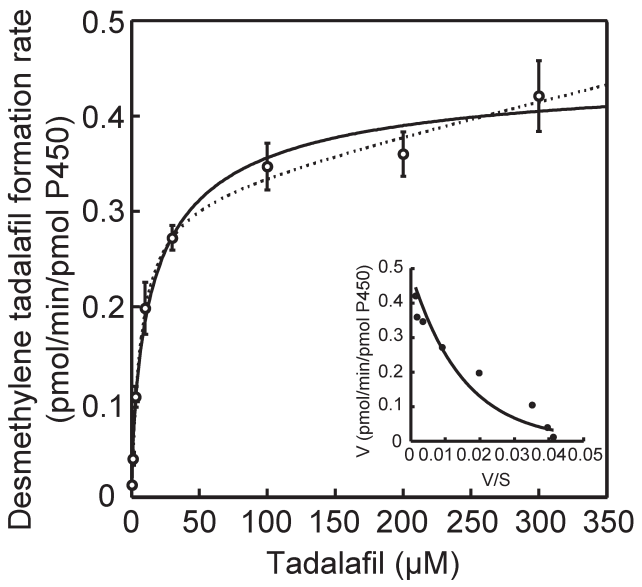

(C)

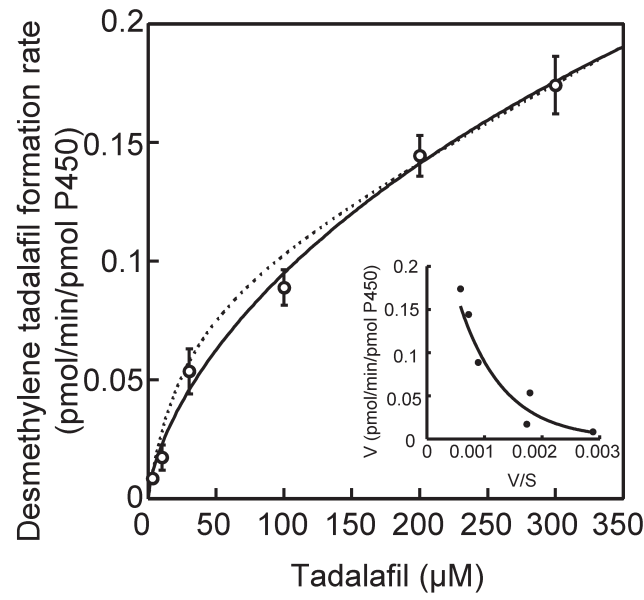

Fig. 3. The Kinetics of Desmethylene Tadalafil Formation from Tadalafil by CYP3A4 (A), CYP3A5 (B), and CYP3A7 Isoforms (C)

Various concentrations of substrate $(0-300 \mu \mathrm{M})$ were incubated with recombinant human CYP3A and NADPH at $37^{\circ} \mathrm{C}$ for $10 \mathrm{~min}$. The velocity (pmol/min/ pmol P450) versus tadalafil concentration was fitted to sigmoidal model (solid line) and two binding sites model (dotted line). The corresponding Eadie-Hofstee plot (velocity versus velocity/substrate concentration) is shown in the inset. Each point represents average \pm S.E. $(n=6)$.

analysis based on a two binding sites model.

In the two binding sites model, the formation process of the initial binding complex with high-affinity $K_{\mathrm{m} 1}$ and lowcapacity $V_{\max 1}$ and that of the secondary binding complex with low-affinity $K_{\mathrm{m} 2}$ and high-capacity $V_{\max 2}$ were assumed to be reaction 1 and reaction 2 , respectively. The kinetics of sildenafil $\mathrm{N}$-demethylation and tadalafil demethylenation cata- 
Table 1. Mean Enzyme Kinetic Parameters of Desalkyl Metabolite Formation of Sildenafil and Tadalafil from CYP3A4, CYP3A5, and CYP3A7 Isoforms with the Hill Equation

\begin{tabular}{|c|c|c|c|c|c|c|}
\hline & \multicolumn{3}{|c|}{ Sildenafil } & \multicolumn{3}{|c|}{ Tadalafil } \\
\hline & CYP3A4 & CYP3A5 & CYP3A7 & CYP3A4 & CYP3A5 & CYP3A7 \\
\hline$V_{\max }(\mathrm{pmol} / \mathrm{min} / \mathrm{pmol} \mathrm{P} 450)$ & $30.7 \pm 11.3$ & $18.9 \pm 6.1$ & $9.92 \pm 0.01$ & $0.658 \pm 0.048$ & $0.467 \pm 0.069$ & $0.872 \pm 0.221$ \\
\hline$K_{\mathrm{m}}(\mu \mathrm{m})$ & $27.7 \pm 7.5$ & $17.4 \pm 3.2$ & $107 \pm 21$ & $5.29 \pm 0.47$ & $7.76 \pm 1.05$ & $171 \pm 68$ \\
\hline$n$ & $0.64 \pm 0.11$ & $0.71 \pm 0.19$ & $0.52 \pm 0.05$ & $0.60 \pm 0.05$ & $0.72 \pm 0.13$ & $0.66 \pm 0.06$ \\
\hline$C L_{\text {int }}(\mu \mathrm{L} / \mathrm{min} / \mathrm{pmol} \mathrm{P} 450)$ & 1.108 & 1.086 & 0.093 & 0.124 & 0.059 & 0.005 \\
\hline
\end{tabular}

The kinetic parameters were determined based on the formation rates of $N$-desmethyl sildenafil and desmethylene tadalafil. Values are expressed as mean \pm S.E.

Table 2. Mean Enzyme Kinetic Parameters of $N$-Desmethyl Sildenafil Formation from CYP3A4, CYP3A5, and CYP3A7 Isoforms with the Two Binding Sites Model

\begin{tabular}{lccc}
\hline \hline & CYP3A4 & CYP3A5 & CYP3A7 \\
\hline Best fit to equation & 3 & 3 & 4 \\
$V_{\max 1}(\mathrm{pmol} / \mathrm{min} / \mathrm{pmol} \mathrm{P450)}$ & $11.0 \pm 2.1$ & $10.8 \pm 2.1$ & $0.452 \pm 0.069$ \\
$K_{\mathrm{m} 1}(\mu \mathrm{M})$ & $15.0 \pm 5.1$ & $13.7 \pm 5.2$ & $5.71 \pm 1.31$ \\
$C L_{\text {int1 }}(\mu \mathrm{L} / \mathrm{min} / \mathrm{pmol} \mathrm{P} 450)$ & 0.733 & 0.788 & 0.079 \\
$V_{\max 2}(\mathrm{pmol} / \mathrm{min} / \mathrm{pmol} \mathrm{P450)}$ & $99.1 \pm 0.0$ & $95.4 \pm 23.1$ & $1.22 \pm 0.05$ \\
$K_{\mathrm{m} 2}(\mu \mathrm{M})$ & $2960 \pm 776$ & $4940 \pm 2530$ & $327 \pm 57$ \\
$C L_{\text {int2 }}(\mu \mathrm{L} / \mathrm{min} / \mathrm{pmol} \mathrm{P450)}$ & 0.033 & 0.019 & 0.004 \\
\hline
\end{tabular}

Values are expressed as mean \pm S.E.

Table 3. Mean Enzyme Kinetic Parameters of $N$-Desmethylene Tadalafil Formation from CYP3A4, CYP3A5, and CYP3A7 Isoforms with the Two Binding Sites Model

\begin{tabular}{lccc}
\hline \hline & CYP3A4 & CYP3A5 & CYP3A7 \\
\hline Best fit to equation & 3 & 4 & 4 \\
$V_{\max 1}(\mathrm{pmol} / \mathrm{min} / \mathrm{pmol} \mathrm{P450)}$ & $0.238 \pm 0.021$ & $0.323 \pm 0.032$ & $0.101 \pm 0.030$ \\
$K_{\mathrm{m} 1}(\mu \mathrm{M})$ & $1.27 \pm 0.19$ & $6.46 \pm 1.66$ & $27.6 \pm 11.4$ \\
$C L_{\text {int1 }}(\mu \mathrm{L} / \mathrm{min} / \mathrm{pmol} \mathrm{P450)}$ & 0.187 & 0.050 & 0.004 \\
$V_{\max 2}(\mathrm{pmol} / \mathrm{min} / \mathrm{pmol} \mathrm{P450)}$ & $0.608 \pm 0.028$ & $0.554 \pm 0.228$ & $0.159 \pm 0.024$ \\
$K_{\mathrm{m} 2}(\mu \mathrm{M})$ & $44.7 \pm 13.0$ & $1620 \pm 776$ & $531 \pm 198$ \\
$C L_{\text {int2 }}(\mu \mathrm{L} / \mathrm{min} / \mathrm{pmol} \mathrm{P450)}$ & 0.014 & $<0.001$ & $<0.001$ \\
\hline
\end{tabular}

Values are expressed as mean \pm S.E.

lyzed by CYP3A isoforms were well fitted to the two binding sites model (Figs. 2, 3), and the estimated kinetic parameters of sildenafil and tadalafil were listed in Table 2 and Table 3, respectively. The $K_{\mathrm{m} 2}$ values for sildenafil $N$-demethylation by CYP3A4/5 and for tadalafil demethylenation by CYP3A5 were much greater than the maximal concentration of substrate tested $(300 \mu \mathrm{M})$. Again, the $K_{\mathrm{m} 2}$ value for tadalafil demethylenation by CYP3A7 was greater than $300 \mu \mathrm{M}$. These results suggested that the contributions of reaction 2 to sildenafil $N$-demethylation by CYP3A4/5 and tadalafil demethylenation by CYP3A5/7 were negligible in our experiment. On the other hand, the $K_{\mathrm{m} 2}$ value for sildenafil $N$-demethylation by CYP3A7 $(327 \mu \mathrm{M})$ was close to $300 \mu \mathrm{M}$, and the $K_{\mathrm{m} 2}$ value for tadalafil demethylenation by CYP3A4 was $44.7 \mu \mathrm{M}$, suggesting that the contributions of reaction 2 to sildenafil $\mathrm{N}$-demethylation by CYP3A7 and tadalafil demethylenation by CYP3A4 were at least in part in our experiment (Tables 2, 3). These results were in line with observations of a biphasic plot obtained for the sildenafil $N$-demethylation by CYP3A7 and the tadalafil demethylenation by CYP3A4 (Figs. 2C, 3A).

\section{DISCUSSION}

The aim of this study was to characterize the kinetics of metabolite formation of the phosphodiesterase type-5 inhibitors sildenafil and tadalafil by CYP3A4, CYP3A5, and CYP3A7 isoforms. This is also the first article documenting the CYP3A-mediated metabolism of tadalafil. Both sildenafil $\mathrm{N}$-demethylation and tadalafil demethylenation were catalyzed by CYP3A4, CYP3A5, and to a lesser extent by CYP3A7 (Figs. 2, 3). The kinetics of desalkyl metabolite formation of the two drugs were well fitted to the Hill equation; however, the Hill coefficients ( $n$ ) suggested CYP3A-mediated negative cooperativity. Next, we analyzed the kinetics with a two binding sites model assuming two reaction steps: reaction 1 with high-affinity and low-capacity metabolism and reaction 2 with low-affinity and high-capacity metabolism. The kinetics of desalkyl metabolite formation were also fitted to the two binding sites model (Figs. 2, 3). The $C L_{\text {int }}$ values of reactions 1 and 2 for sildenafil $N$-demethylation were 0.733 and $0.033 \mu \mathrm{L} /$ $\mathrm{min} / \mathrm{pmol} \mathrm{P450}$ for CYP3A4, 0.788 and $0.019 \mu \mathrm{L} / \mathrm{min} / \mathrm{pmol}$ P450 for CYP3A5, and 0.079 and $0.004 \mu \mathrm{L} / \mathrm{min} / \mathrm{pmol} \mathrm{P} 450$ for 
CYP3A7, respectively (Table 2). The $C L_{\text {int }}$ values of reaction 1 and 2 for tadalafil demethylenation were 0.187 and $0.014 \mu \mathrm{L} /$ $\mathrm{min} / \mathrm{pmol}$ P450 for CYP3A4, 0.050 and $<0.001 \mu \mathrm{L} / \mathrm{min} / \mathrm{pmol}$ P450 for CYP3A5, and 0.004 and $<0.001 \mu \mathrm{L} / \mathrm{min} / \mathrm{pmol} \mathrm{P} 450$ for CYP3A7, respectively (Table 3).

Korzekwa et $a .^{23)}$ evaluated atypical cytochrome $\mathrm{P} 450$ kinetics with a two binding sites model in which the enzyme can bind to two substrate molecules simultaneously, and demonstrated that the two binding sites model can be used to describe activation, autoactivation, partial inhibition, substrate inhibition, and biphasic saturation curves for the cytochrome P450 enzymes. In the present study, we tried to understand the mechanisms for CYP3A-mediated negative cooperativity with a hypothesized two distinct steps of metabolite formation (reactions 1, 2). For reaction 1, a first substrate (S) binds to CYP3A enzyme (E) and forms a binary complex (ES: one substrate and one enzyme), which is catalytically active, but has a relatively low maximal rate $\left(V_{\max 1}\right)$. For reaction 2 , a second substrate (S) binds to CYP3A to form a ternary complex (SES: two substrates and one enzyme) and has a higher maximal rate $\left(V_{\max 2}\right)$. The enzyme kinetic parameters (Tables 2,3 ) based on the two binding sites model in which $V_{\max 1}<V_{\max 2}$ and $K_{\mathrm{m} 1}<K_{\mathrm{m} 2}$ were compatible with the results that a biphasic saturation profile was clearly observed in the sildenafil $\mathrm{N}$ demethylation by CYP3A7 (Fig. 2C) and the tadalafil demethylenation by CYP3A4 (Fig. 3A). No apparent biphasic saturation profile was observed in the sildenafil $\mathrm{N}$-demethylation by CYP3A4/5 (Figs. 2A, B) and the tadalafil demethylenation by CYP3A5/7 (Figs. 3B, C) in cases where the $K_{\mathrm{m} 2}$ value was much greater than the range of substrate concentrations tested (Tables 2, 3).

$\mathrm{Ku}$ et $a{ }^{11)}$ demonstrated that the catalytic efficiency $\left(C L_{\text {int }}=V_{\max }\right.$ /apparent $\left.K_{\mathrm{m}}\right)$ of the CYP3A5 isoform for sildenafil $N$-demethylation was similar to the CYP3A4 isoform by using CYP3A supersomes. The reported mean values of $K_{\mathrm{m}}$ for sildenafil $N$-demethylation (15.0 and $14.7 \mu \mathrm{M}$, for CYP3A4 and CYP3A5, respectively) were comparable to those for reaction $1\left(K_{\mathrm{m} 1}\right)$ in the present study (Table 2), whereas the reported mean values of $V_{\max }$ for sildenafil $(1.00$ and $1.38 \mathrm{pmol} / \mathrm{min} /$ pmol P450, for CYP3A4 and CYP3A5, respectively) were significantly lower than those for reaction $1\left(V_{\max 1}\right)$ in the present study (Table 2). The experimental materials used in our study were similar to those in the study by $\mathrm{Ku}$ et al., ${ }^{11)}$ but the difference in experimental design may reflect the discrepancy between the two studies. That is, in our experiment, CYP3A supersomes were first mixed with NADPH and then metabolic reactions were started by addition of the substrate (sildenafil or tadalafil). On the other hand, the reaction was initiated by adding an NADPH-generating system following a 5 min preincubation of CYP3A enzymes with sildenafil in the study by $\mathrm{Ku}$ et $a l .{ }^{11)}$ Interestingly, they demonstrated that the metabolism of sildenafil exhibited substrate inhibition kinetics. ${ }^{11)}$ Actually, the metabolism of sildenafil exhibited substrate inhibition kinetics and resulted in the decreased $V_{\max }$ values in our preliminary experiment using CYP3A4 supersomes (data not shown). In this case, the reaction was initiated by adding NADPH following a 5-min preicubation of CYP3A4 enzyme with sildenafil. Thus, the difference between NADPH and NADPH-generating system is unlikely to be responsible for the decrease in $V_{\max }$. Several attempts have been made to understand the mechanism of substrate inhibition by using the two binding sites model. ${ }^{24,25)}$ It is assumed that the two sites are either neighboring or at a distance within the active site and that one site is favorable for oxidation while the other site is less favorable or nonproductive. ${ }^{24)}$ When the substrate binds to the inhibitory site, the inhibited ternary complex (SES) is less capable of converting substrate to product than binary complex (ES). ${ }^{24)}$ Thus, if both binding sites of CYP3A are occupied with substrates during the preincubation step, the velocity of enzymatic reactions per protein $\left(V_{\max }\right)$ will be underestimated by loss in activity. On the other hand, tadalafil is reported to be a mechanism-based inactivator having a methylenedioxyphenyl functional group in its structure. ${ }^{26)}$ Tadalafil inactivated 1'-OH-midazolam formation in a time- and concentration-dependent manner with mean values for $k_{\text {inact }}$ (the formation rate constant of the inactive complex with the enzyme) and $K_{\mathrm{I}}$ (the dissociation constant for the inactivator) of $0.21 \mathrm{~min}^{-1}$ and $12 \mu \mathrm{M}$, respectively. ${ }^{26)}$ Although the mechanism-based inactivation (MBI) will reduce the active enzyme pool, the incubation time employed for tadalafil was $10 \mathrm{~min}$ and the consumption of the drug at all concentrations was less than $3 \%$ under the conditions of the present experiment. Our experimental protocol was designed to minimize MBI effects; however, further studies will be required to clarify the causal relationship between properties of MBI and negative cooperative kinetics.

We previously evaluated the variability in the pharmacokinetics of tadalafil in 23 children with $\mathrm{PAH}^{27)}$ The peak plasma concentrations of tadalafil ranged from 0.33 to $2.91 \mu \mathrm{M}(1.17 \pm 0.64 \mu \mathrm{M})$ in children with a mean dose of $0.97 \mathrm{mg} \cdot \mathrm{kg}^{-1} \cdot \mathrm{d}^{-1}$, and a large interindividual difference in the protein binding rate (84.6-99.4\%) was observed. ${ }^{27)}$ On the other hand, it was reported that the plasma concentration of sildenafil at $1 \mathrm{~h}$ after oral administration was $0.23 \pm 0.18 \mu \mathrm{M}$ in 3 children with $\mathrm{PAH}{ }^{28)}$ The unbound fraction of sildenafil was reported to be about $2-4 \%$ in humans. ${ }^{9)}$ Assuming that only the unbound form of the drug is able to reach the specific binding sites of CYP3A molecules, reaction 2 of each drug was unlikely to be responsible for in vivo metabolism because of its low affinity for substrates. We also approximated the velocity of the CYP3A-mediated desalkyl reaction based on the kinetic parameters listed in Tables 2 and 3. When the sildenafil concentration is $0.23 \mu \mathrm{M}$, the velocities of drug $N$-demethylation by CYP3A4, CYP3A5, and CYP3A7 are calculated to be $0.166,0.178$, and $0.018 \mathrm{pmol} / \mathrm{min} / \mathrm{pmol} \mathrm{P} 450$, respectively. When the tadalafil concentration is $1.17 \mu \mathrm{M}$, the velocities of drug demethylenation by CYP3A4, CYP3A5, and CYP3A7 are calculated to be $0.120,0.050$, and $0.004 \mathrm{pmol} /$ $\mathrm{min} / \mathrm{pmol} \mathrm{P} 450$, respectively. These results suggested that the relative contributions of CYP3A5 and CYP3A7 to the total clearance of tadalafil were smaller than those of sildenafil.

Recently, it was reported that the effect of genetic polymorphisms of CYP3A5 on the disposition of CYP3A substrates differs from drug to drug. That is, Loh et al. ${ }^{29)}$ evaluated the effect of CYP3A5 genotypes on the pharmacokinetics of two CYP3A substrates, tacrolimus and cyclosporine, and demonstrated that CYP3A5 genotypes had a significant impact on tacrolimus concentration-to-dose (C/D) ratios, but there were no correlations between cyclosporine $\mathrm{C} / \mathrm{D}$ ratios and CYP3A5 genotypes. In addition, the capability for CYP3A7 in the metabolism of sildenafil and tadalafil was lower than those for CYP3A4 and CYP3A5 (Table 1), which was in line with 
the previous reports by Williams et al. ${ }^{30)}$ They demonstrated that the $K_{\mathrm{m}}$ values for CYP3A7 tended to be greater than those for CYP3A4 and CYP3A5 in the metabolism of several CYP3A substrates such as midazolam, triazolam, tamoxifen, etc. However, considering that CYP3A7 is assumed to play an important role in the fetal stage and that idiopathic and/or heritable PAH (IPAH and/or HPAH) were epidemiologically more common in women, the possibility of exposure of PDE5 inhibitors to fetus cannot be ruled out. Further studies may be needed to confirm the effects of genetic polymorphisms of CYP3A5 or the developmental change in CYP3A7 activity on drug disposition, and to develop a dosage guideline regarding the transition from sildenafil to tadalafil in patients with PAH.

In conclusion, we demonstrated that the catalytic activity and relative contribution of the three CYP3A isoforms for major metabolite formations were not the same for sildenafil and tadalafil. The kinetic analysis with a two binding sites model have had success in describing the observations presented here. The present study may provide the basis not only for understanding the metabolic properties of the two PDE5 inhibitors, but also for one possible explanation of the mechanism of CYP3A-mediated negative cooperativity.

Acknowledgments This work was supported in part by Grants-in-Aid for Scientific Research from the Japan Society for the Promotion of Sciences (JSPS KAKENHI Grant Number 24590182).

Conflict of Interest The authors declare no conflict of interest.

\section{REFERENCES}

1) Rybalkin SD, Yan C, Bornfeldt KE, Beavo JA. Cyclic GMP phosphodiesterases and regulation of smooth muscle function. Circ. Res., 93, 280-291 (2003)

2) Lewis GD, Semigran MJ. Type 5 phosphodiesterase inhibition in heart failure and pulmonary hypertension. Curr. Heart Fail. Rep., 1, 183-189 (2004)

3) Takatsuki S, Calderbank M, Ivy DD. Initial experience with tadalafil in pediatric pulmonary arterial hypertension. Pediatr. Cardiol., 33, 683-688 (2012).

4) Ghofrani HA, Voswinckel R, Reichenberger F, Olschewski H, Haredza P, Karadas B, Schermuly RT, Weissmann N, Seeger W, Grimminger F. Differences in hemodynamic and oxygenation responses to three different phosphodiesterase-5 inhibitors in patients with pulmonary arterial hypertension: a randomized prospective study. J. Am. Coll. Cardiol., 44, 1488-1496 (2004).

5) Nichols DJ, Muirhead GJ, Harness JA. Pharmacokinetics of sildenafil after single oral doses in healthy male subjects: absolute bioavailability, food effects and dose proportionality. Br. J. Clin. Pharmacol., 53 (Suppl. 1), 5S-12S (2002).

6) Forgue ST, Patterson BE, Bedding AW, Payne CD, Phillips DL, Wrishko RE, Mitchell MI. Tadalafil pharmacokinetics in healthy subjects. Br. J. Clin. Pharmacol., 61, 280-288 (2006).

7) Jackson G, Rosen RC, Kloner RA, Kostis JB. The second Princeton consensus on sexual dysfunction and cardiac risk: new guidelines for sexual medicine. J. Sex. Med., 3, 28-36, discussion, 36 (2006).

8) Shlobin OA, Brown AW, Weir N, Ahmad S, Lemma M, Nathan SD. Transition of PH patients from sildenafil to tadalafil: feasibility and practical considerations. Lung, 190, 573-578 (2012).

9) Muirhead GJ, Wilner K, Colburn W, Haug-Pihale G, Rouviex B. The effects of age and renal and hepatic impairment on the phar- macokinetics of sildenafil. Br. J. Clin. Pharmacol., 53 (Suppl. 1), 21S-30S (2002).

10) Hyland R, Roe EG, Jones BC, Smith DA. Identification of the cytochrome P450 enzymes involved in the $N$-demethylation of sildenafil. Br. J. Clin. Pharmacol., 51, 239-248 (2001).

11) Ku HY, Ahn HJ, Seo KA, Kim H, Oh M, Bae SK, Shin JG, Shon $\mathrm{JH}$, Liu KH. The contributions of cytochromes P450 3A4 and 3A5 to the metabolism of the phosphodiesterase type 5 inhibitors sildenafil, udenafil, and vardenafil. Drug Metab. Dispos., 36, 986-990 (2008).

12) Forgue ST, Phillips DL, Bedding AW, Payne CD, Jewell H, Patterson BE, Wrishko RE, Mitchell MI. Effects of gender, age, diabetes mellitus and renal and hepatic impairment on tadalafil pharmacokinetics. Br. J. Clin. Pharmacol., 63, 24-35 (2007).

13) Ring BJ, Gillespie JS, Mullen JH, Wrighton SA. Identification of the human cytochrome P450 (CYP) responsible for the formation of the major oxidative metabolite of tadalafil (Cialis). Drug Metab. Rev., 36 (S1), 70 (2004).

14) Phillips DL, Smith RL, Patterson BE, Parker N, Mitchell M, Wheeler WJ, Watkins VS, Barbuch RJ. Metabolism and excretion of tadalafil in healthy men after oral administration of $100 \mathrm{mg}$ [14C]tadalafil. AAPS J., 6, W5308 (2004).

15) Lacroix D, Sonnier M, Moncion A, Cheron G, Cresteil T. Expression of CYP3A in the human liver-evidence that the shift between CYP3A7 and CYP3A4 occurs immediately after birth. Eur. J. Biochem., 247, 625-634 (1997).

16) Stevens JC, Hines RN, Gu C, Koukouritaki SB, Manro JR, Tandler PJ, Zaya MJ. Developmental expression of the major human hepatic CYP3A enzymes. J. Pharmacol. Exp. Ther., 307, 573-582 (2003).

17) Sim SC, Edwards RJ, Boobis AR, Ingelman-Sundberg M. CYP3A7 protein expression is high in a fraction of adult human livers and partially associated with the CYP3A7*1C allele. Pharmacogenet. Genomics, 15, 625-631 (2005).

18) Kim J, Ji H, Kim SJ, Lee HW, Lee SS, Kim DS, Yoo M, Kim WB, Lee HS. Simultaneous determination of sildenafil and its active metabolite UK-103,320 in human plasma using liquid chromatographytandem mass spectrometry. J. Pharm. Biomed. Anal., 32, 317-322 (2003).

19) Al-Ghazawi M, Tutunji M, Aburuz S. Simultaneous determination of sildenafil and $N$-desmethyl sildenafil in human plasma by highperformance liquid chromatography method using electrochemical detection with application to a pharmacokinetic study. J. Pharm. Biomed. Anal., 43, 613-618 (2007).

20) Vos RM, Chahbouni A, Sinjewel A, Swart EL. Quantitative analysis of sildenafil and desmethylsildenafil in human serum by liquid chromatography-mass spectrometry with minimal sample pretreatment. J. Chromatogr. B Analyt. Technol. Biomed. Life Sci., 876, 283-287 (2008).

21) Farthing CA, Farthing DE, Koka S, Larus T, Fakhry I, Xi L, Kukreja RC, Sica D, Gehr TW. A simple and sensitive HPLC fluorescence method for determination of tadalafil in mouse plasma. $J$. Chromatogr. B Analyt. Technol. Biomed. Life Sci., 878, 2891-2895 (2010).

22) Miller GP, Guengerich FP. Binding and oxidation of alkyl 4-nitrophenyl ethers by rabbit cytochrome P450 1A2: evidence for two binding sites. Biochemistry, 40, 7262-7272 (2001).

23) Korzekwa KR, Krishnamachary N, Shou M, Ogai A, Parise RA, Rettie AE, Gonzalez FJ, Tracy TS. Evaluation of atypical cytochrome P450 kinetics with two-substrate models: evidence that multiple substrates can simultaneously bind to cytochrome P450 active sites. Biochemistry, 37, 4137-4147 (1998).

24) Lin Y, Lu P, Tang C, Mei Q, Sandig G, Rodrigues AD, Rushmore TH, Shou M. Substrate inhibition kinetics for cytochrome P450catalyzed reactions. Drug Metab. Dispos., 29, 368-374 (2001).

25) Reed MC, Lieb A, Nijhout HF. The biological significance of substrate inhibition: a mechanism with diverse functions. BioEssays, 
32, 422-429 (2010).

26) Ring BJ, Patterson BE, Mitchell MI, Vandenbranden M, Gillespie J, Bedding AW, Jewell H, Payne CD, Forgue ST, Eckstein J, Wrighton SA, Phillips DL. Effect of tadalafil on cytochrome P450 3A4-mediated clearance: studies in vitro and in vivo. Clin. Pharmacol. Ther., 77, 63-75 (2005).

27) Kohno H, Ichida F, Hirono K, Ozawa S, Yoshimura N, Nakamura T, Akita C, Ishida K, Taguchi M. Plasma concentrations of tadalafil in children with pulmonary arterial hypertension. Ther. Drug Monit., 36, 576-583 (2014).

28) Karatza AA, Bush A, Magee AG. Safety and efficacy of sildenafil therapy in children with pulmonary hypertension. Int. J. Cardiol., 100, 267-273 (2005).

29) Loh PT, Lou HX, Zhao Y, Chin YM, Vathsala A. Significant impact of gene polymorphisms on tacrolimus but not cyclosporine dosing in Asian renal transplant recipients. Transplant. Proc., 40, 1690-1695 (2008).

30) Williams JA, Ring BJ, Cantrell VE, Jones DR, Eckstein J, Ruterbories K, Hamman MA, Hall SD, Wrighton SA. Comparative metabolic capabilities of CYP3A4, CYP3A5, and CYP3A7. Drug Metab. Dispos., 30, 883-891 (2002). 